\title{
Clinical values of common biomarkers for efficacy monitoring of antibiotics in early-onset neonatal sepsis
}

\author{
Chunmei Liu, Ying Zhang, Yanyan Shang, Chengzhi Fang, Qi He, Lili Xie \\ Department of Neonatology, Renmin Hospital of Wuhan University \& Hubei General Hospital, Wuhan, China \\ Contributions: (I) Conception and design: C Liu; (II) Administrative support: C Liu; (III) Provision of study materials or patients: All authors; (IV) \\ Collection and assembly of data: All authors; (V) Data analysis and interpretation: All authors; (VI) Manuscript writing: All authors; (VII) Final \\ approval of manuscript: All authors. \\ Correspondence to: Chunmei Liu. Department of Neonatology, Renmin Hospital of Wuhan University and Hubei General Hospital, Wuhan 430060, \\ China. Email: liuchunmei123@126.com.
}

Background: To investigate the clinical values of the common biomarkers including blood routine (BRt), C-reactive protein (CRP), serum amyloid A (SAA) and procalcitonin (PCT) for efficacy monitoring of antibiotics in early-onset neonatal sepsis (EONS).

Methods: The clinical and laboratory data of 78 neonates with confirmed EONS in the neonatal intensive care unit (NICU) of our center from July 1, 2019 to June 30, 2020 were retrospectively analyzed. All the subjects were treated with cefotiam $(50 \mathrm{mg} / \mathrm{kg} \mathrm{q} 12 \mathrm{~h})$ and augmentin $(30 \mathrm{mg} / \mathrm{kg} \mathrm{q} 12 \mathrm{~h})$ within 12 hours after birth. Blood samples were collected 0-12 hours after birth for blood culture, measurements of B-Rt, CRP and SAA. Subsequently, blood sampling was performed at intervals of 12-24, 24-48, 48-96, and 96-144 hours for measurements of B-Rt, CRP, SAA and PCT. Statistical analyses were performed in the SPSS 20.0 software package. $P$ value of $<0.05$ was considered statistically significant.

Results: WBC count showed no significant change among different intervals (12-24, 24-48, 48-96, and 96-144 hours); in contrast, NEU\%, CRP, SAA and PCT significantly differed across all intervals. SAA had sensitivities of $75.86 \%, 93.1 \%, 44.83 \%$, and $3.45 \%$, respectively; specificities of $100 \%$ across all intervals; and AUCs of $0.879(\mathrm{P}<0.0001), 0.966(\mathrm{P}<0.0001), 0.724(\mathrm{P}<0.0001)$, and 0.500, respectively $(\mathrm{P}=1)$. PCT had sensitivities of $100 \%, 100 \%, 79.31 \%$, and $51.72 \%$, respectively; specificities of $100 \%$ across all intervals; and AUCs of 1 ( $\mathrm{P}<0.0001), 1(\mathrm{P}<0.0001), 0.793(\mathrm{P}<0.0001)$, and 0.517 ( $\mathrm{P}>0.8551)$, respectively.

Conclusions: WBC count, NEU\% and CRP showed no clinical significance for any intervals for efficacy monitoring of antibiotic treatment. SAA and PCT had similar monitoring values at 12-24 and 24-48 hours. SAA is thus more valuable than PCT for efficacy monitoring of antibiotics at the 48-96 and even at the 96-144 hours intervals in EONS.

Keywords: C-reactive protein (CRP); serum amyloid protease A (SAA); procalcitonin (PCT); total white blood cell (WBC) count; neutrophil percentage (NEU\%); early-onset neonatal sepsis (EONS); efficacy monitoring

Submitted Sep 03, 2020. Accepted for publication Oct 20, 2020.

doi: $10.21037 / \mathrm{tp}-20-326$

View this article at: http://dx.doi.org/10.21037/tp-20-326

\section{Introduction}

Early-onset neonatal sepsis (EONS) in the neonatal intensive care unit (NICU) is highly fatal and can lead to serious long-term sequelae. Timely diagnosis and appropriate antibiotic use are particularly important for lowering EONS-related mortality and morbidity $(1,2)$.
The gold-standard blood culture technique requires at least 24-48 hours and low positive yields. The clinical manifestations vary considerably and are nonspecific, which makes the diagnosis of EONS difficult and predisposes to excessive antibiotic use. This has resulted in the early, broad, and prolonged application of antibiotics in 
suspected cases with negative blood culture results (3-6). The excessive or irrational use of antibiotics not only leads to high hospitalization expenditures (7), but also may cause changes in the normal gut flora, induction of multi-drug-resistant bacteria, and increased risks of serious complications including invasive candidiasis, necrotizing enterocolitis, delayed sepsis, and even death $(8,9)$. In recent decades have seen emergence of multiple putative biomarkers for efficacy monitoring of antibiotics EONS. These include surface markers, acute phase reactants, cytokines, and chemokines. These objective biomarkers such as B-Rt, CRP, SAA, PCT and IL-6 are most widely used in the clinical. B-Rt is the most basic clinical biomarker, despite the development of other biomarkers in recent years, CRP remains the most commonly use diagnostic and monitoring indicator for sepsis, with a specificity of $94.8 \%$ and a sensitivity of $67.1 \%$ (10). Meanwhile, PCT is a polypeptide consisting of 116 amino acids. Studies have found that PCT has the highest negative predictive value $(87-100 \%)$ for severe bacterial infections in newborns $(11,12)$. Finally, SAA is an apolipoprotein mainly synthesized by the liver. It has high accuracy for the early detection of infections in neonates, with a specificity of $95 \%$ and a sensitivity of $82 \%$, and it is negatively correlated with neonatal mortality (13). Cytokines and chemokines such as interleukin-6 (IL-6) has been deemed unsuitable because of its short half-life (14). Meanwhile, bacterial DNA test has low specificity and expensive, and has not been widely used in clinical practice.

With the goal of identifying biomarkers capable of guiding antibiotic adjustment and discontinuation, our study retrospectively explored the features of B-Rt, CRP, SAA and PCT during the treatment of EONS. Different from previous studies, this study mainly compared the specificity and sensitivity of these most widely used conventional biomarkers in the clinical for efficacy monitoring. We present the following article in accordance with the STARD reporting checklist (available at http://dx.doi.org/10.21037/ tp-20-326).

\section{Methods}

\section{Subjects}

A total of 78 patients being cared for in the NICU of our center from July 1, 2019 to June 30, 2020 were selected for this retrospective study. All cases were admitted to the NICU within 2 hours after birth in the Obstetrics Department, and their gestational ages ranged from 28 to
34 weeks. All subjects met the following diagnostic criteria for EONS (15): (I) the suspected cases had any of the following features within 3 days of birth: (i) abnormal clinical manifestations; (ii) a mother with chorioamnionitis; or (iii) premature rupture of membranes (PROM) $\geq 18$ hours before the onset of contractions. If there were no abnormal clinical manifestations, blood culture was negative, and two consecutive blood non-specific tests (within a 24-hour interval) showed fewer than two positive results, the possibility of sepsis was ruled out. (II) A clinical diagnosis was made if a patient had abnormal clinical manifestations and met any of the following criteria: (i) blood non-specific tests showing two or more positive results; (ii) cerebrospinal fluid test revealing purulent meningitis; or (iii) the presence of bacterial DNA in the blood. (III) A diagnosis of EONS was confirmed if there were typical clinical manifestations of sepsis and positive findings in blood/cerebrospinal fluid (or other sterile cavity fluid) cultures.

The study follows the tenets of the Declaration of Helsinki (as revised in 2013) and was approved by the Ethics Committee of People's Hospital of Wuhan University (no registry number is available as no relevant project funding for this study was provided). The Ethics Committee of our school agreed, but no number was given because there was no relevant project funding for this article). The guardians of all the children in our department gave informed consent.

\section{Measurements}

A retrospective study was conducted on 78 patients with confirmed EONS. All the subjects were treated with cefotiam $(50 \mathrm{mg} / \mathrm{kg} \mathrm{q} 12 \mathrm{~h}$ ) and augmentin $(30 \mathrm{mg} / \mathrm{kg} \mathrm{q} 12 \mathrm{~h})$ within 12 hours after birth. The medicines were switched to third- or later-generation broad-spectrum antibiotics (meropenem, imipenem-cilastatin sodium, and others) within 24 hours, and the treatments lasted for 5 days or more. Automated blood culture was performed (BacT/ ALERT3D60, Bio Mérieux), along with the use of a 24-hour early warning system. A preliminary culture report was generated 3 days (72 hours) after culture for bacteria, and a final culture report was obtained at day 5 (120 hours). B-Rt was completed on an automatic blood analyzer, CRP and SAA were measured by enzyme-linked immunosorbent method (ELISA: Booster Biological Technology Co. Ltd. Wuhan, China), and PCT was determined by immunoluminescence method (E601, Roche Cobas). All the tests were completed in our clinical laboratory.

According to the clinical routine monitoring of blood 
Table 1 WBC count, NEU\%, CRP, SAA and PCT at different intervals

\begin{tabular}{lccccc}
\hline Interval & WBC $\left(\times 10^{9} / \mathrm{L}\right)[\mathrm{M}(\mathrm{P} 25, \mathrm{P} 75)]$ & $\mathrm{NEU} \%(\bar{x} \pm \mathrm{S})$ & $\mathrm{CRP}(\mathrm{mg} / \mathrm{L})[\mathrm{M}(\mathrm{P} 25, \mathrm{P} 75)]$ & $\mathrm{SAA}(\mathrm{mg} / \mathrm{L})[\mathrm{M}(\mathrm{P} 25, \mathrm{P} 75)]$ & $\mathrm{PCT}(\mathrm{ng} / \mathrm{mL})[\mathrm{M}(\mathrm{P} 25, \mathrm{P} 75)]$ \\
\hline 0-12 h & $8.77(6.38,14.21)$ & $55.20 \pm 16.67$ & $0.5(0.5,0.5)$ & $5(5,5)$ & - \\
$12-24 \mathrm{~h}$ & $9.25(7.18,15.86)$ & $75.84 \pm 9.13$ & $12.35(5.60,28.33)$ & $67.06(5.54,161.05)$ & $25.10(12.16,79.05)$ \\
$24-48 \mathrm{~h}$ & $8.99(7.00,12.35)$ & $71.21 \pm 13.35$ & $19.45(11.03,32.01)$ & $104.73(44.28,144.77)$ & $26.96(8.39,50.25)$ \\
$48-96 \mathrm{~h}$ & $9.12(7.29,12.29)$ & $54.18 \pm 14.40$ & $6.97(2.51,12.39)$ & $5(5,10.90)$ & $2.57(0.40,10.61)$ \\
$96-144 \mathrm{~h}$ & $12.86(9.66,15.12)$ & $43.58 \pm 12.37$ & $1.81(0.93,3.92)$ & $5(5,5)$ & $0.26(0.12,0.52)$ \\
Statistic & $\mathrm{H}=8.225$ & $\mathrm{~F}=28.325$ & $\mathrm{H}=80.397$ & $\mathrm{H}=79.802$ & $\mathrm{H}=72.076$ \\
P value & 0.084 & $<0.001$ & $<0.001$ & $<0.001$ & $<0.001$
\end{tabular}

WBC, white blood cell; NEU\%, neutrophils; CRP, C-reactive protein; SAA, serum amyloid A; PCT, procalcitonin.

routine and the characteristics of CRP, SAA and PCT in the process of inflammatory response and the actual clinical monitoring of the course of disease in neonates, monitoring was conducted every $12-24$ hours within 72 hours after birth, and every 24-48 hours after the condition was stabilized after 72 hours. Because PCT can be altered by physiological factors such as labor factors and postnatal hunger, the inflammatory response within 12-24 hours (16), so PCT monitoring begins 12 hours after birth. We detected WBC, NEU\%, CRP, SAA and PCT using the following protocol: the first session of blood specimen collection, blood culture, and determination of B-Rt, CRP and SAA was completed within 12 hours after birth for all subjects, for whom the detection of B-Rt, CRP and SAA was performed using the same blood specimen; in the other intervals (12-24, 24-48, 48-96, and 96-144 hours), B-Rt, CRP, SAA and PCT were determined using the same blood samples. In this study, all the cases were from the obstetrics department of pathology in our hospital, and the conditions of the included cases were strictly controlled. In order to reduce the variability of the results as much as possible, all the test biomarkers were applied with the same specimen at the same time.

\section{Data analysis}

Diagnostic performance of WBC count, CRP, SAA and PCT were analyzed by non-parametric statistical methods, while NEU\% was analyzed by chi-squared test in the SPSS 20.0 software package (IBM, Armonk, NY, USA). The receiver operating characteristic (ROC) curves were drawn by using the MedCalc 18.2.1 software (MedCalc Software bv, Ostend, Belgium) to compare the diagnostic sensitivities and specificities. A $\mathrm{P}$ value of $<0.05$ was considered statistically significant.

\section{Results}

\section{General data}

All the cases in the EONS group met the diagnostic criteria for EONS (15). The WBC count, NEU\%, CRP, SAA and PCT at different intervals are summarized in Table 1. The roles of WBC count, NEU\%, CRP, SAA and PCT in monitoring the treatment responses in patients are summarized in Table 2.

\section{Levels of inflammatory biomarkers at different intervals}

The WBC count, NEU\%, CRP, SAA and PCT at different intervals are summarized. After tests for normality and homogeneity of variance, the diagnostic performance of WBC count, CRP, SAA and PCT were analyzed by nonparametric statistical methods, while NEU\% was analyzed by chi-squared test in the SPSS 20.0 software package (Table 1 ). WBC count showed no significant change across the such different intervals (12-24, 24-48, 48-96, and 96144 hours); NEU\%, CRP, SAA and PCT showed significant change across all intervals (Table 1).

\section{Areas under the ROC curve of each inflammation biomarkers at different intervals}

The sensitivity and specificity of each marker were calculated by using the MedCalc software (version 18.2.1) (Table 2). For the intervals of 12-24, 24-48, 48-96, and 96-144 hours, WBC had sensitivities of $82.76 \%, 75.86 \%$, 
Table 2 Performance of WBC count, NEU\%, CRP, SAA and PCT in monitoring the treatment responses in EONS patients

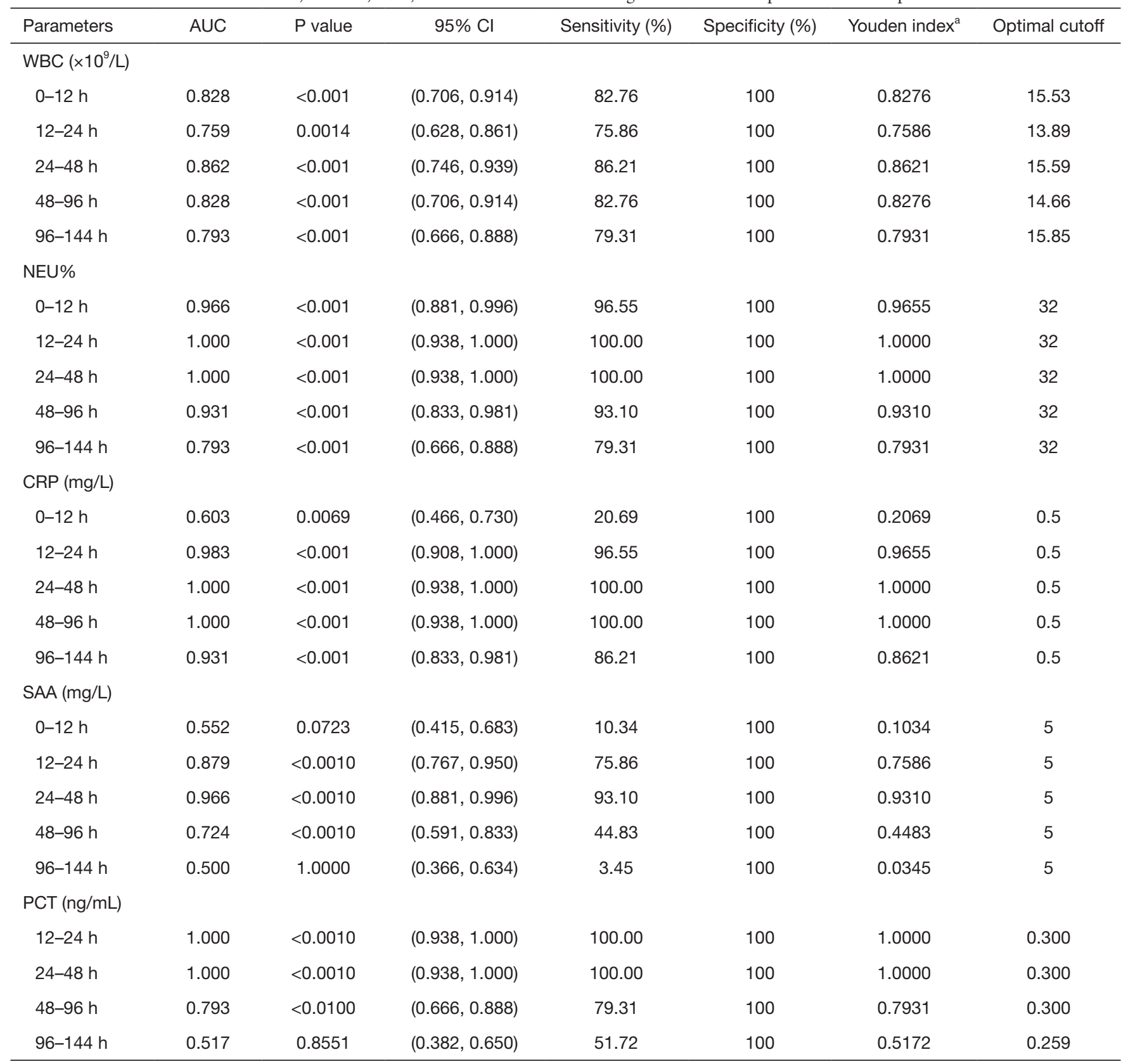

a, Youden Index = Specificity + Sensitivity - 1. WBC, white blood cell; NEU\%, neutrophils; CRP, C-reactive protein; SAA, serum amyloid A; $\mathrm{PCT}$, procalcitonin; EONS, early-onset neonatal sepsis.

$86.21 \%, 82.76 \%$, and $79.31 \%$, respectively; specificities of $100 \%$ across all intervals; optimal cutoffs of $15.53,13.89$, $15.59,14.66$, and $15.85 \times 10^{9} / \mathrm{L}$, respectively; and AUCs of $0.828,0.759,0.862,0.828$, and 0.793 , respectively (all $\mathrm{P}<0.01$ ). NEU\% had sensitivities of $96.55 \%, 100 \%$, $100 \%, 93.1 \%$, and $79.31 \%$, respectively; specificities of $100 \%$ across all intervals; optimal cutoffs of $32 \%$ across all intervals; and AUCs of 0.966, 1, 1, 0.931, and 0.793 , respectively (all $\mathrm{P}<0.01$ ). CRP had sensitivities of $20.69 \%, 96.55 \%, 100 \%, 100 \%$, and $86.21 \%$, respectively; specificities of $100 \%$ across all intervals; optimal cutoffs of $0.5 \mathrm{mg} / \mathrm{L}$ across all intervals; and AUCs of 0.603 , $0.983,1,1$, and 0.931 , respectively. SAA had sensitivities of $75.86 \%, 93.1 \%, 44.83 \%$, and $3.45 \%$, respectively; 


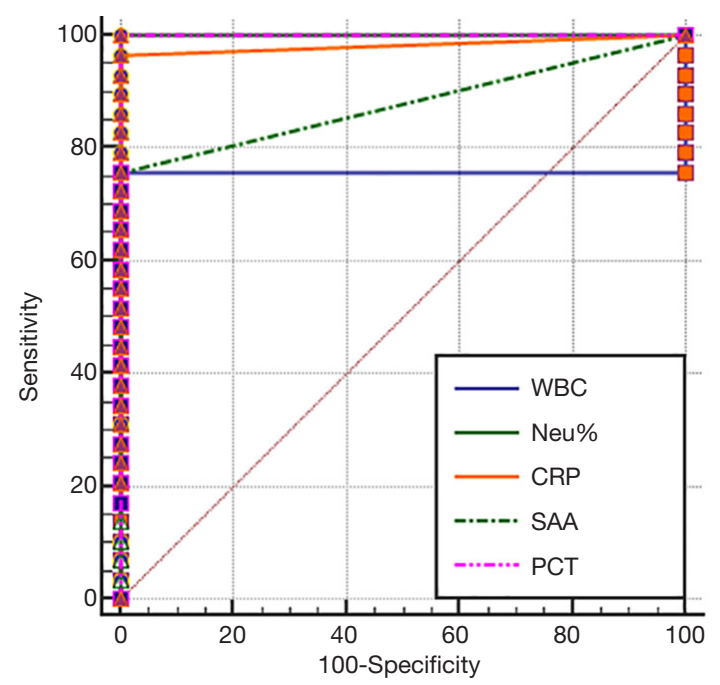

Figure 1 AUC analysis results of the values of WBC count, NEU\%, CRP, SAA and PCT in monitoring treatment response at the 12-24 hours interval. AUC, areas under the ROC curve; WBC, white blood cell; NEU\%, neutrophils; CRP, C-reactive protein; SAA, serum amyloid A; PCT, procalcitonin.

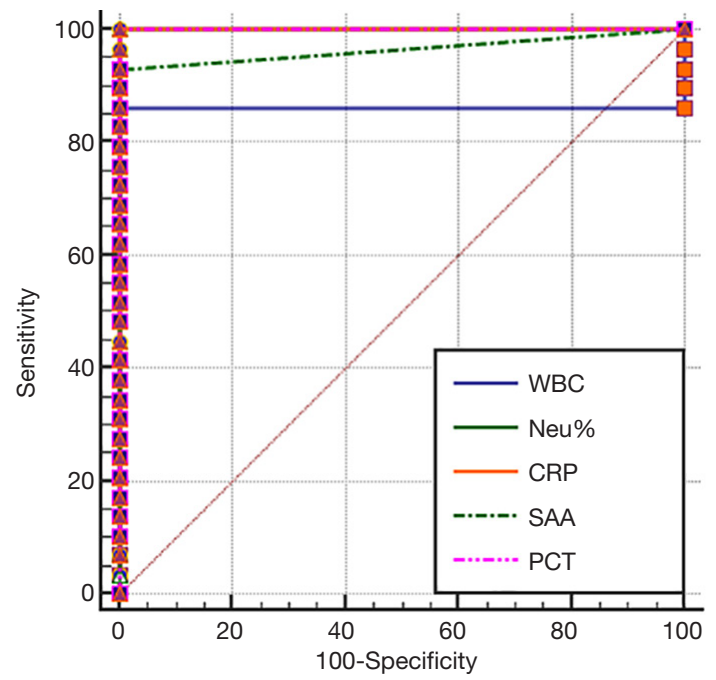

Figure 2 AUC analysis results of WBC count, NEU\%, CRP, SAA and PCT values for monitoring treatment response at the 2448 hours interval. AUC, areas under the ROC curve; WBC, white blood cell; NEU\%, neutrophils; CRP, C-reactive protein; SAA, serum amyloid A; PCT, procalcitonin.

specificities $100 \%$ across all intervals; optimal cutoffs of $5 \mathrm{mg} / \mathrm{L}$ across all intervals; and AUCs of $0.879(\mathrm{P}<0.0001)$, $0.966(\mathrm{P}<0.0001), 0.724(\mathrm{P}<0.0001)$, and $0.500(\mathrm{P}=1)$, respectively. PCT had sensitivities of $100 \%, 100 \%, 79.31 \%$, and $51.72 \%$, respectively; specificities of $100 \%$ across all intervals; optimal cutoffs of $0.3,0.3,0.3$, and $0.259 \mathrm{ng} / \mathrm{mL}$, respectively; and AUCs of $1(\mathrm{P}<0.0001), 1(\mathrm{P}<0.0001), 0.793$ $(\mathrm{P}<0.0001)$, and $0.517(\mathrm{P}>0.8551)$, respectively.

\section{Values of different inflammatory biomarkers for efficacy monitoring in EONS patients at different intervals}

The area under the ROC curve (AUC), which is used for evaluating diagnostic performance, ranged between 0.5 and 1. The diagnostic performance is regarded as low when AUC is $0.5-0.7$, intermediate when AUC is $0.7-0.9$, and high when AUC is 0.9 or higher. AUC $=0.5$ indicates a worthless test. The sensitivity and specificity of each marker were calculated by using the MedCalc software (version 18.2.1) (Table 2). The AUC analysis results of the values of WBC count, NEU\%, CRP, SAA and PCT in monitoring the treatment response in EONS patients are shown in Figures 1-4.

As shown in Tables 1 and 2 and in Figures 1-4, WBC count had a moderate diagnostic value at each interval; CRP had a high diagnostic value at each interval(with an AUC above 0.9); SAA had a high diagnostic value at 24 48 hours (with an AUC of above 0.9), a moderate diagnostic value at the intervals of 12-24 hours and 48-96 hours (with AUCs being 0.7-0.9), but had no diagnostic value at the interval of 96-144 hours (with an AUC of 0.5); PCT had a high diagnostic value at intervals of 12-24 and 24-48 hours (with an AUC of above 0.9), a moderate diagnostic value at 48-96 hours (with AUCs of 0.7-0.9), but had no diagnostic value at 96-144 hours (with an AUC of 0.517). The diagnostic sensitivities of WBC count, NEU\%, CRP, SAA and PCT ranged from $86.21 \%$ to $100 \%$ at $12-24$ hours and $24-48$ hours; were $82.76 \%, 93.1 \%, 100 \%$, $44.83 \%$, and $79.31 \%$ at $48-96$ hours, respectively; and were $79.31 \%, 79.31 \%, 86.21 \%, 3.45 \%$, and $51.72 \%$ at $96-$ 144 hours, respectively.

Therefore, WBC count, NEU\%, and CRP had no value in monitoring treatment response at any of the intervals; SAA and PCT had similar values for efficacy monitoring at 12-24 and 24-48 hours; SAA was more valuable than PCT in monitoring treatment response at 48-96 hours and even at $96-144$ hours.

\section{Discussion}

EONS is a common diagnosis among neonates admitted to the NICU. While it is a highly fatal condition that requires 


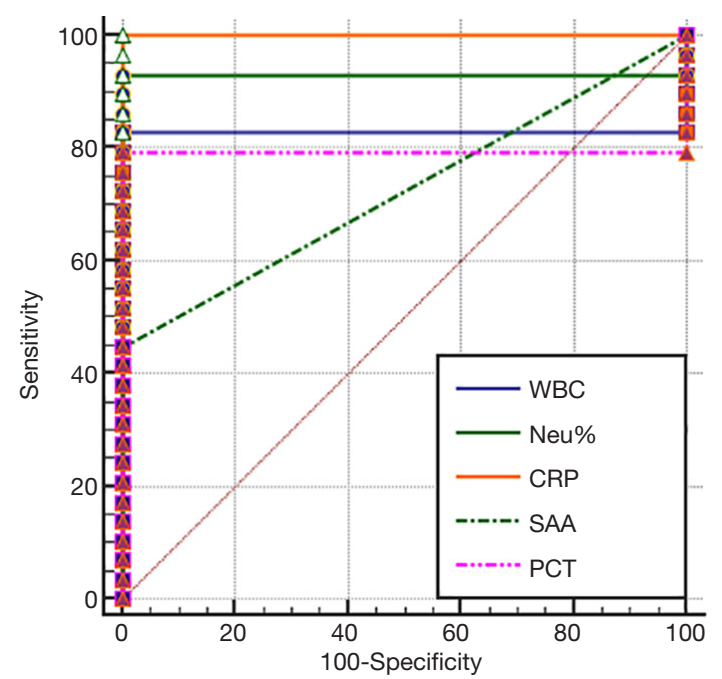

Figure 3 AUC analysis results of the WBC count, NEU\%, CRP, SAA and PCT values in monitoring treatment response at the 48 96 hours interval. AUC, areas under the ROC curve; WBC, white blood cell; NEU\%, neutrophils; CRP, C-reactive protein; SAA, serum amyloid A; PCT, procalcitonin.

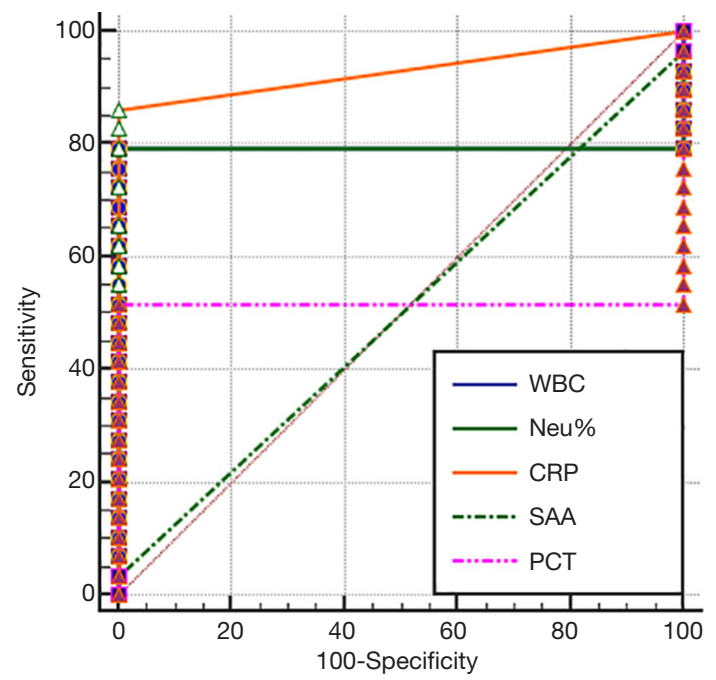

Figure 4 AUC analysis results of the WBC count, NEU\%, CRP, SAA and PCT values in monitoring treatment response at the 96-144 hours interval. AUC, areas under the ROC curve; WBC, white blood cell; NEU\%, neutrophils; CRP, C-reactive protein; SAA, serum amyloid A; PCT, procalcitonin.

immediate treatment, blood culture is time-consuming and has a low positivity rate. Thus, biomarkers including B-Rt, CRP, SAA and PCT are often used to assist the clinical diagnosis of EONS, which may lead to antibiotic overuse. Furthermore, these conventional biomarkers are also used for deciding the initiation, adjustment, and discontinuation of antibiotic therapy. Research has shown that the duration and intensity of antibiotic use are longer/higher in China than in Western countries (17). In addition, perinatal antibiotic exposure increases the risk of drug resistance and is associated with a series of shortand long-term risks (7-9). Thus, timely discontinuation of antibiotic therapy is particularly important. A small number of international studies have used PCT as a monitoring biomarker for discontinuing antibiotics in the ICU and the PICU; however, to our knowledge, no study has yet described the use of CRP, SAA and PCT for monitoring treatment response in EONS neonates. Our current study was designed to observe the changes of CRP, SAA and PCT during antibiotic therapy for EONS and to explore the sensitivities and specificities of these biomarkers in monitoring treatment response, with an attempt to offer new evidence for guiding early antibiotic use in EONS infants.

The most commonly used biomarkers in the diagnosis of infection include CRP, SAA and PCT (18-20). Recent studies have demonstrated that PCT has a higher diagnostic sensitivity and specificity compared with conventional CRP. However, PCT is affected by physiological factors and stress in the perinatal period, which makes PCT less valuable within the first 12 hours after delivery. In contrast, the SAA level rapidly decreases only if the infection is well controlled (13). Therefore, we tried to detect the levels of these biomarkers at different intervals in EONS neonates and to explore their monitoring values during antibiotic treatment.

Clinically, after the use of early-generation antibiotics within 12 hours after birth, the patients still had severe clinical symptoms; after the use of latergeneration antibiotics, the infants were still at the peak of inflammation. Although SAA and PCT had similar high diagnostic and sensitivity values at 24-48 and 4896 hours intervals because of their similar AUC, the AUC and sensitivity of SAA was much more remarkably decreased than those of the PCT, indicating that although the disease was still serious, the antibiotics had conferred a therapeutic effect, thus offered a chance for deescalating or even discontinuing antibiotic therapy window period during at 48-96 hours after birth. Furthermore, the AUC values of SAA and PCT were 0.5 and 0.517 at $96-$ 144 hours respectively, indicating that the AUCs of both PCT and SAA dramatically dropped and approached 
normal levels; meanwhile, the sensitivity values of SAA and PCT were $3.45 \%$ and $51.72 \%$ respectively, indicating that SAA could better reflect the disease improvement and thus is more suitable as an indicator for discontinuing antibiotic therapy. We consequently speculated that because the extent of SAA concentration change is closely related to the severity of inflammation. Therefore during acute phase of inflammation, SAA rises rapidly, but when the neonate is better after the antibiotic treatment has taken effect, SAA also declines rapidly, so it can be sensitive to reflect the level of antibiotic efficacy and changes in the condition of neonates. Lateral results showed that the changes were closely related to the severity of the disease, SAA has obvious advantages over PCT/CRP in efficacy monitoring. Thus, SAA is particularly much more valuable for the monitoring of antibiotic use than PCT: (I) at the intervals of 48 96 hours after birth, SAA can raise a prompt alarm for deescalating or even discontinuing antibiotic use, which is of great significance for reducing the intensity of antibiotic use and the degree of antibiotic use; (II) on the other hand it plays an active role in evaluating efficacy monitoring at 96144 hours, alerting the physicians to discontinue or further de-escalate antibiotic use, which is of much more great significance for shortening antibiotic use or for lowering the intensity of antibiotic treatment in the clinical.

Based on the features of inflammation biomarkers during sepsis, we measured B-Rt, CRP, SAA and PCT levels at different time intervals to avoid human error. Due to the need for continuous monitoring of biomarkers for the newborns in this sample, strict inclusion criteria were adopted in this retrospective study. So although the sample size was relatively small, the wide range of cases in our department compensated for this shortage to some extent, further multi-center studies are needed to supplement this research found that multi-center supplement.

In summary, this study analyzed the roles of B-Rt, CRP, SAA and PCT in monitoring treatment response during the antibiotic treatment of EONS. While WBC count, NEU\%, and CRP have little value in monitoring treatment response in different intervals, SAA and PCT exhibited moderate value in treatment response monitoring, with SAA having a higher monitoring value than PCT. When applied in clinical settings, SAA may help to reduce the intensity and duration of antibiotic use in the NICU.

\section{Acknowledgments}

Funding: None.

\section{Footnote}

Reporting Checklist: The authors have completed the STARD reporting checklist. Available at http://dx.doi.org/10.21037/ tp-20-326

Data Sharing Statement: Available at http://dx.doi. org/10.21037/tp-20-326

Conflicts of Interest: All authors have completed the ICMJE uniform disclosure form (available at http://dx.doi. org/10.21037/tp-20-326). The authors have no conflicts of interest to declare.

Etbical Statement: The authors are accountable for all aspects of the work in ensuring that questions related to the accuracy or integrity of any part of the work are appropriately investigated and resolved. The study follows the tenets of the Declaration of Helsinki (as revised in 2013) and was approved by the Ethics Committee of People's Hospital of Wuhan University (no registry number is available as no relevant project funding for this study was provided). The Ethics Committee of our school agreed, but no number was given because there was no relevant project funding for this article). The guardians of all the children in our department gave informed consent.

Open Access Statement: This is an Open Access article distributed in accordance with the Creative Commons Attribution-NonCommercial-NoDerivs 4.0 International License (CC BY-NC-ND 4.0), which permits the noncommercial replication and distribution of the article with the strict proviso that no changes or edits are made and the original work is properly cited (including links to both the formal publication through the relevant DOI and the license). See: https://creativecommons.org/licenses/by-nc-nd/4.0/.

\section{References}

1. Stocker M, van Herk W, El Helou S, et al. Procalcitoninguided decision making for duration of antibiotic therapy in neonates with suspected early-onset sepsis: a multicentre, randomised controlled trial (NeoPIns). Lancet 2017;390:871-81.

2. Weiss SL, Fitzgerald JC, Balamuth F, et al. Delayed antimicrobial therapy increases mortality and organ dysfunction duration in pediatric sepsis. Crit Care Med 2014;42:2409-17. 
3. Schulman J, Profifit J, Lee HC, et al. Variations in neonatal antibiotic use. Pediatrics 2018;142:e20180115.

4. Greenberg RG, Chowdhury D, Hansen NI, et al. Prolonged duration of early antibiotic therapy in extremely premature infants. Pediatr Res 2019;85:994-1000.

5. Schulman J, Dimand RJ, Lee HC, et al. Neonatal intensive care unit antibiotic use. Pediatrics 2015;135:826-33.

6. Flannery DD, Ross RK, Mukhopadhyay S, et al. Temporal trends and center variation in early antibiotic use among premature infants. JAMA Network Open 2018;1:e180164.

7. Carr D, Barnes EH, Gordon A, et al. Effect of antibiotic use on antimicrobial antibiotic resistance and lateonset neonatal infections over 25 years in an Australian tertiary neonatal unit. Arch Dis Child Fetal Neonatal Ed 2017;102:F244-50.

8. Cantey JB, Patel SJ. Antimicrobial stewardship in the NICU. Infect Dis Clin North Am 2014;28:247-61.

9. Mukhopadhyay S, Sengupta S, Puopolo KM. Challenges and opportunities for antibiotic stewardship among preterm infants. Arch Dis Child Fetal Neonatal Ed 2019;104:F327-32.

10. Hofer N, Zacharias E, Müller W, et al. An update on the use of C-reactive protein in early-onset neonatal sepsis: current insights and new tasks. Neonatology 2012;102:25-36.

11. van Rossum AM, Wulkan RW, Oudesluys-Murphy AM. Procalcitonin as an early marker of infection in neonates and children. Lancet Infect Dis 2004;4:620-30.

12. Vouloumanou EK, Plessa E, Karageorgopoulos DE, et al. Serum procalcitonin as a diagnostic marker for neonatal

Cite this article as: Liu C, Zhang Y, Shang Y, Fang C, He Q, Xie L. Clinical values of commonly used biomarkers for monitoring antibiotic use in neonates with early-onset neonatal sepsis. Transl Pediatr 2020;9(5):669-676. doi: 10.21037/tp-20-326 sepsis: a systematic review and meta-analysis. Intensive Care Med 2011;37:747-62.

13. Liu C, Fang C, Xie L. Diagnostic utility of procalcitonin as a biomarker for late-onset neonatal sepsis. Transl Pediatr 2020;9:237-42.

14. Wang Q, Zhou X, Yang L, et al. Gentiopicroside (GENT) protects against sepsis induced by lipopolysaccharide (LPS) through the NF- $\kappa \mathrm{B}$ signaling pathway. Ann Transl Med. 2019;7:731.

15. Subspecialty Group of Neonatology, the Society of Pediatric, Chinese Medical Association; Professional Committee of Infectious Diseases, Neonatology Society, Chinese Medical Doctor Association. Zhonghua Er Ke Za Zhi 2019;57:252-7.

16. Chiesa C, Pacifico L, Natale F, et al. Fetal and early neonatal interleukin-6 response. Cytokine 2015;76:1-12.

17. Lu C, Liu Q, Yuan H, et al. Implementation of the Smart Use of Antibiotics Program to Reduce Unnecessary Antibiotic Use in a Neonatal ICU: A Prospective Interrupted Time-Series Study in a Developing Country. Crit Care Med 2019;47:e1-e7.

18. Simonsen KA, Anderson-Berry AL, Delair SF, et al. Earlyonset neonatal sepsis, Clin Microbiol Rev 2014;27:21-47.

19. Sack GH Jr. Serum Amyloid A (SAA) Proteins. Subcell Biochem 2020;94:421-36.

20. Chauhan N, Tiwari S, Jain U. Potential biomarkers for effective screening of neonatal sepsis infections: An overview. Microb Pathog 2017;107:234-42.

(English Language Editor: J. Gray) 